\title{
The Information Systems Interoperability Maturity Model (ISIMM): Towards Standardizing Technical Interoperability and Assessment within Government
}

\author{
STEFANUS Van $\operatorname{Staden}^{1}$ and JAMESON Mbale ${ }^{2}$ \\ ${ }^{1}$ Office of the Prime Minister, Government Buildings, Windhoek, Namibia \\ sstaden@opm.gov.na \\ ${ }^{2}$ University of Namibia, Centre of Excellence in Telecommunications, Department of Computer Science, Private Bag \\ 13301, Windhoek,Namibia \\ mbalej@yahoo.com
}

\begin{abstract}
To establish and implement a workable e-Government, all possible and relevant stakeholders' systems need to be inter-connected in such away that the hardware, software and data are interoperable. Thus, interoperability is the key to information exchange and sharing among the heterogeneous systems. In view of this, the paper introduces the Information Systems Interoperability Maturity Model (ISIMM) that defines the levels and degree of interoperability sophistication that an organisation's Information Systems will progress through. ISIMM focuses more on detailed technical aspects of interoperability that allows data to be exchanged and shared within an information system environment. In this way, it provides the practical means of assessing technical interoperability between information system pairs, groups or clusters and it facilitates a model to measure the maturity and compliancy levels of interoperability information systems.
\end{abstract}

Index Terms - ISIMM, e-Government, benchmark, interoperability, interoperability maturity model, maturity level, compliance level, technical interoperability, assessment, information systems and data.

\section{INTRODUCTION}

Electronic Government (e-Government) reflects the final vision for Governments that will allow them to undergo enormous modernization and reorganisation [1].

To achieve the aims of e-Government, there is a need to connect Government (i.e., G2G, G2C, G2B and $\mathrm{G} 2 \mathrm{E}$ ) internally and externally to its stakeholders. The key concept that the connected Government is built on is that of interoperability [2]. Interoperable systems working in a seamless and coherent manner across public service sectors hold the key to providing better public services and making the public sector more competitive and service-oriented.

Before a government start with comprehensive e-integration initiatives, it needs to understand it's current and desired state of interoperability. With the aid of an interoperability maturity model, a Government can determine its current interoperability capability and identify its desired interoperability capability and sophistication. By knowing where you are and where you want to be, a strategy can be devised to move towards a desired state of interoperability.

In line with the above mentioned, the Government of Namibia embarked upon an exercise in 2011 to set its desired state of interoperability and establish its current state of interoperability. The exercise primary focused on interoperability between information systems at a more technical level. As part of the exercise, a number of existing interoperability maturity models where assessed for suitability. The next section states the problems that were identified during the maturity model assessment process.

A number of interoperability models have been developed of which some are reviewed in Section 2 . From the models studied it was found that they did not address technical interoperability maturity and assessment to a satisfactory level for software architects. Most of these models were also complex and focused on a broader range of interoperability characteristics.

There was a need for a maturity model that focused in more detail on the technical aspects of information systems interoperability and met the requirements of a specific domain (e.g., public service).

The organisation of this paper is as follows: section II looks at what other scholars have done. Section III covers some objectives of this work, where as, section IV outlines the methodology used in this paper. Section $\mathrm{V}$ explains the information system interoperability maturity model and section VI gives the highlights of applying the ISIMM. Section VII concludes this study.

\section{LITERATURE REVIEW}

Most Maturity models describe the stages of progress or evolution through which systems, 
processes or organisations progress [3].

A variety of interoperability maturity models have been developed, each adopting a unique vocabulary to express their characterisation of interoperability capability maturity. These maturity models address specific problem domains.

In 1993 the Levels of Information Systems Interoperability (LISI) project was initiated by the C4ISR Integration Task Force to address the specific requirements of C4I (Command, Control, Computer, Communication and Intelligence) domain. The outcome of the LISI project was a LISI reference model and process for defining, assessing and certifying the degree of interoperability required or achieved between organisations or information systems. The LISI Interoperability Maturity Model of [4] defines five stages of increasing levels of sophistication regarding information system interaction and the ability of the system to exchange and share information and services such as: (1) Enterprise, (2) Domain,(3) Functional, (4) Connected, and (5) Isolated.

The Organisational Interoperability Maturity Model for C2 was proposed by [3] and serves to compliment the LISI reference model by extending it into the area of organisational interoperability. The Organisational Interoperability Maturity Model for C2 defines five levels of organisational maturity of which each level is defined by one or more primary enabling attributes. The C2 maturity levels are: (1) Unified, (2) Combined, (3) Collaborative, (4) Ad-hoc, and (5) Independent.

The Capability Maturity Matrix Integration (CMMI) was proposed by [5] as a process improvement approach that can be used to guide process improvement across entities. CMMI helps to integrate organisational functions, set process improvement goals and priorities, provide guidance, and serve as a reference for appraising processes. CMMI consists of 22 process areas with capability or maturity levels.

[6] proposed the Government Interoperability Model Matrix (GIMM) that can be used by organisations to assess their current e-Government Interoperability status in respect to interoperability readiness and performance. The GIMM defines five different sets of organisational interoperability maturity levels, where each level corresponds to a different interoperability level for a set of Interoperability Attributes (IA). The organisational interoperability maturity levels defined in GIMM are closely aligned to the CMMI reference model and to LISI. The GIMM maturity levels are the following: (1) Independent, (2) Ad-hoc, (3) Collaborative, (4) Integrated and (5) Unified.

The interoperability maturity models reviewed (i.e., LISI, C2, CMMI, and GIMM) define very similar interoperability maturity levels with the main differences between the models being their focus and the manner in which they rate interoperability. The models are partial models that deal with some aspects of the enterprise interoperability domain. The models further were not developed to a satisfactory level to measure explicit potential. An interoperability maturity model covering all areas of concern and aspects of enterprise and e-Government interoperability is still missing.

\section{OBJECTIVES}

The Information System Interoperability Maturity Model (ISIMM) was developed in-line with the following objectives:

1. Serve as interoperability maturity standard and guide for information system pairs, groups and sectors at a technical level

2. Provide the means to benchmark technical interoperable information system pairs, groups and sectors against the maturity standards defined; and

3. Should be easy to understand and use.

\section{METHODOLOGY}

In developing ISIMM, a study was conducted that made use of a qualitative research approach in which explorative research was combined with case study research methods. The population sample for the study consisted of IT Managers and IT Staff from the Government of Namibia who was responsible for operationalized Information Systems. Data was obtained by using both purposive and snowball sampling methods. The study provided the background and specification that was used to formulate the maturity levels and assessment framework.

The ISIMM was tested with 24 information system pairs from seven (7) Namibia government organisations.

\section{INFORMATION SYSTEM INTEROPERABILTY MATURITY MODEL}

To assess the degree of interoperability between Information Systems, a more practical Information Systems' Interoperability Maturity Model (ISIMM) was developed to meet the objectives stated previously. The ISIMM was derived from the theories of LISI and GIMM and specifically focuses in more detailed on the technical aspects of interoperability that would allow data to be shared and exchanged within an information systems environment.

Specially, ISIMM in Figure 1 defines the levels and degree of interoperability sophistication that an organisation's Information Systems will progress through. The levels of ISIMM provide a structured and systematic approach for assessing and measuring Information Systems' interoperability maturity. In addition to exploring the complexities of interoperability, ISIMM provides the means to attain a deeper understanding of Information Systems' interoperability that will help to promote and establish an interoperable systems environment within government. 


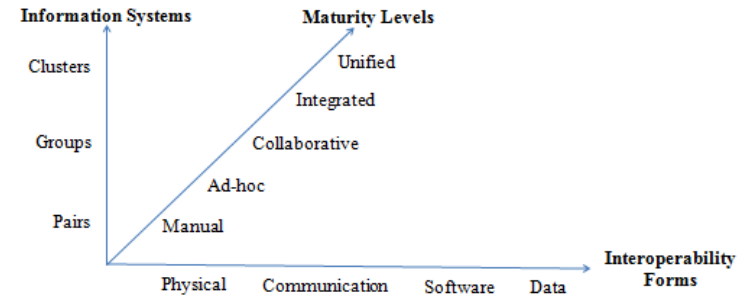

Figure 1. Information system interoperability model (ISIMM)

\subsection{Technical interoperability layers}

The ISIMM focus on technical interoperability of information systems is in the following areas:

1. Data Interoperability: Data interoperability denotes the ability of different software from heterogeneous systems to understand the syntactical and semantic meaning of data from different data models through the use of common data models, mappings and structures.

2. Software Interoperability: Refers to the ability of different software used by organisations to work together in exchanging and sharing of data by solving the differences between them.

3. Communication

Interoperability: Communication interoperability denotes the ability of systems to connect and communicate through common protocols.

4. Physical Interoperability: Physical interoperability is the ability of different computer hardware, network devices and peripherals to work in a connected way.

\subsection{ISIMM maturity model}

The interoperability maturity levels of ISIMM as depicted in Figure 2, define the progression of an interoperable environment from a high disparate Information Systems' environment to a high common integrated and shared Information Systems' environment. This categorises a move from a low level to a high level of Information Systems' environment interoperability.

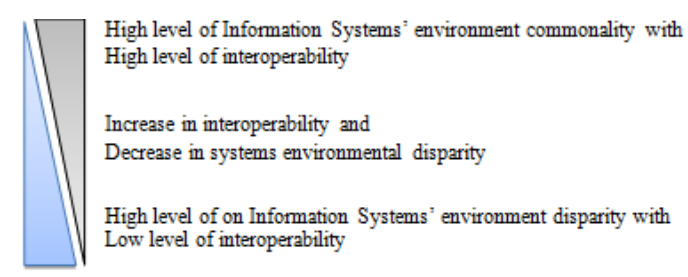

Figure 2. Information systems' interoperability maturity transition

The maturity interoperability computing environment levels of ISIMM as depicted in Figure 1 are defined below:
Level 1 - Manual: Information Systems are not connected and data sharing between systems are through manual means.

Level 2 - Ad-Hoc: Basic data sharing of non-standardised data take place through simple electronic means with other organisations. Applications and databases are separated and data is not shared between organisations. Data are exchanged between systems in a point-to-point manner on an ad-hoc basis.

Level 3 - Collaborative: At this level a wider connection to legacy systems are facilitated. Basic collaboration takes place at a program level between independent applications in a distributed manner. Logical data models are shared and used in the data exchange process. Minimal common functions exist, applications and databases are separated and data is not shared.

Level 4 - Integrated: Data in the integrated stage are shared to some extend and data is exchanged between independent applications using shared domain based data models. Collaboration is at an advanced domain level. Integration of services or systems is being implemented between organisations.

Level 5- Unified: In the unified stage, data and applications are fully shared and distributed between organisations. Collaboration is at an advanced enterprise level with organisations interoperating on continues basis through high quality services. Data have a common interpretation and are based on a common exchange model. Front and back office systems are fully interoperable. Processes are also automated at this level.

\subsection{ISIMM compliance level}

ISIMM is visualized in the Information Systems' Interoperability Maturity and Functional Compliancy Matrix in Table 1. 
Table 1. Information systems' interoperability maturity and functional compliancy matrix

\begin{tabular}{|c|c|c|c|c|c|c|}
\hline Code & $\begin{array}{l}\text { Interoperability } \\
\text { Layers and Attributes }\end{array}$ & $\begin{array}{c}\text { l:Manual } \\
\text { (1.4) } \\
(2) \\
(2)\end{array}$ & $\begin{array}{l}\text { L } \\
\text { 2:Ad-hoc } \\
\text { (5.7) } \\
(6) \\
\end{array}$ & $\begin{array}{l}\text { Degrees of Inter } \\
\text { 3:Codlaborative } \\
\text { (8.10) } \\
(9)\end{array}$ & 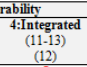 & $\begin{array}{c}\text { 5:Uninied } \\
(14 s) \\
(15) \\
(15)\end{array}$ \\
\hline $\mathrm{D}$ & Data Interoperability & $\frac{12}{1}$ & $\frac{1}{2}$ & & & \\
\hline & $\begin{array}{l}\text { Common Data } \\
\text { Presentation Format }\end{array}$ & $\mathrm{E}$ & E & E & $E$ & $E$ \\
\hline 2 & $\begin{array}{l}\text { Shared Meta-Content } \\
\text { (Data about data }\end{array}$ & & & $\mathrm{E}$ & $\mathrm{E}$ & E \\
\hline 3 & Common Data Model & & & $E$ & E & $E$ \\
\hline 4 & 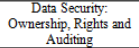 & & E & E & E & E \\
\hline $\begin{array}{l}5 \\
5 \\
\end{array}$ & $\begin{array}{l}\text { Shared Data } \\
\text { Softrare }\end{array}$ & & & & $E$ & $E$ \\
\hline & Interoperability & 1 & 2 & 3 & 5 & 8 \\
\hline 1 & $\begin{array}{l}\text { N-Tier Common } \\
\text { Interoperablity } \\
\text { Architecture }\end{array}$ & & & & $\mathrm{E}$ & E \\
\hline$\frac{2}{3}$ & $\begin{array}{l}\text { Data Exchange Services } \\
\text { Divertor Services }\end{array}$ & $\bar{E}$ & $\bar{E}$ & $\bar{E}$ & $\frac{E}{E}$ & $\frac{E}{E}$ \\
\hline 4 & $\begin{array}{l}\text { Common Naming } \\
\text { Services }\end{array}$ & & & & & $\mathrm{E}$ \\
\hline 5 & $\begin{array}{l}\text { Discovery Services } \\
\text { Schitef }\end{array}$ & & & $E$ & $E$ & E \\
\hline & $\begin{array}{l}\text { Commonon Wortiow } \\
\text { Services }\end{array}$ & & & & & $\mathrm{E}$ \\
\hline 7 & $\begin{array}{l}\text { Security Management } \\
\text { Services }\end{array}$ & & $\mathrm{E}$ & E & E & $\mathrm{E}$ \\
\hline 8 & $\begin{array}{l}\text { Shared Applications } \\
\text { Commutiction }\end{array}$ & & & & & $E$ \\
\hline & $\begin{array}{l}\text { Communication } \\
\text { Interoperability }\end{array}$ & 0 & 1 & 1 & 1 & 1 \\
\hline 1 & $\begin{array}{c}\text { Common } \\
\text { Communication } \\
\text { Protocols }\end{array}$ & & E & E & E & E \\
\hline $\mathrm{P}$ & $\begin{array}{c}\text { Physical } \\
\text { Interoperability }\end{array}$ & 0 & 1 & 1 & 1 & 1 \\
\hline 1 & $\begin{array}{l}\text { Shared Commuminication } \\
\text { Networkt }\end{array}$ & & $\mathrm{E}$ & $\mathrm{E}$ & E & $E$ \\
\hline
\end{tabular}

The Information Systems' interoperability maturity levels is categorised in the matrix within four dimensions, each consisting of a vector of attributes. Each of these dimensions corresponds to the interoperability layers of: (1) Data Interoperability, (2) Software Interoperability, (3) Communication Interoperability and (4) Physical Interoperability.

The baseline interoperability functional compliance requirements for each attribute of every interoperability layer are indicated in Table 1 with an ' $\mathrm{E}$ ' (i.e., Expected).

Table 2. Information systems' interoperability maturity ratings matrix

\begin{tabular}{|c|c|c|c|c|c|c|}
\hline \multirow[b]{2}{*}{ Code } & \multirow{2}{*}{$\begin{array}{l}\text { Interoperability Layers } \\
\text { and Attributes }\end{array}$} & \multicolumn{5}{|c|}{ Levels/Degrees of Interoperability } \\
\hline & & $\begin{array}{c}\text { 1:Manual } \\
(1-4)\end{array}$ & $\begin{array}{l}\text { 2:Ad-hoc } \\
\text { (5-7) } \\
(6)\end{array}$ & $\begin{array}{c}\text { 3:Collaborative } \\
\left(\begin{array}{c}(8-10) \\
(9)\end{array}\right.\end{array}$ & $\begin{array}{l}\text { 4:IItegrated } \\
\begin{array}{c}(11-13) \\
(12)\end{array}\end{array}$ & \begin{tabular}{|c|} 
5:Unified \\
$(14>)$ \\
$(15)$
\end{tabular} \\
\hline D & Data Ir & 1 & $\frac{1}{2}$ & 4 & 5 & \\
\hline s & Software & 1 & 2 & 3 & 5 & 8 \\
\hline $\mathrm{C}$ & $\begin{array}{l}\text { Communication } \\
\text { Interoperability }\end{array}$ & 0 & 1 & 1 & 1 & 1 \\
\hline$P$ & Physical Interoperability & $e^{0}$ & , & 1 & 1 & 1 \\
\hline
\end{tabular}

The range scores for each maturity level/degree are indicated in Table 2 at the top of the table (e.g., 1-4, 5-7, $8-10,11-13,14>)$. The overall compliancy rating is indicated below the range scores. The expected level/degree of compliance for each interoperability layer (i.e., D, S, C, and P) is defined in Table 2. These scores were derived from Table 1 for the compliancy attributes marked for each interoperability layer.

\subsection{ISIMM measurements}

ISIMM provides a model to measure the maturity and compliancy level of interoperability Information Systems either in pairs, groups or clusters.

The Information Systems' Interoperability Maturity and Functional Compliancy Matrix (see Table 1) and Information Systems' Interoperability Maturity Ratings Matrix (see Table 2) services as the instrument to assess both the compliancy as well as the degree of interoperability of an Information System or between Information Systems. A maturity rating is found by identifying the number of compliant attributes for an Information System or pair of Information Systems and comparing the number found with the rating ranges indicated in Table 3. The maturity layers defined should serve as additional guidance in establishing the level of maturity attained.

Table 3. ISIMM interoperability measures

\begin{tabular}{|c|c|c|}
\hline Metric Type & Measures & Code \\
\hline \multirow{3}{*}{ Compliancy } & Below the expected level & $\mathrm{B}$ \\
\cline { 2 - 3 } & Expected level & $\mathrm{E}$ \\
\cline { 2 - 3 } & Above the expected level & $\mathrm{A}$ \\
\hline \multirow{3}{*}{$\begin{array}{c}\text { Computing Environment } \\
\text { Maturity Levels }\end{array}$} & Unified & 5 \\
\cline { 2 - 3 } & Integrated & 4 \\
\cline { 2 - 3 } & Collaborative & 3 \\
\cline { 2 - 3 } & Ad-hoc & 2 \\
\hline \multirow{3}{*}{ Interoperability Layers } & Manual & 1 \\
\cline { 2 - 3 } & Data & $\mathrm{D}$ \\
\cline { 2 - 3 } & Software & $\mathrm{C}$ \\
\cline { 2 - 3 } & Communication & $\mathrm{P}$ \\
\cline { 2 - 3 } & Physical & $1-8$ \\
\hline Interoperability Attributes & Sub Levels defimed from ' 1 ' to ' 8 ' & \\
\hline
\end{tabular}

A scorecard is presented in Table 4 to record the level and form of Information Systems' interoperability compliancy between Information Systems.

Table 4: Information systems' interoperability scorecard

\begin{tabular}{|c|c|c|c|c|}
\hline Information Systems & System 1 & System 2 & System 3 & System N \\
\hline System 1 & eg., IE & & & \\
\hline System 2 & & & & \\
\hline System 3 & & & & \\
\hline System N & & & & \\
\hline
\end{tabular}

The scorecard in Table 4 is a matrix that consists of Information Systems represented in both the rows and columns. Each row and column intersection indicates the system-to-system interoperability as pairs of values from the interoperability metrics defined in Table 3 .

Using the compliancy matrix and scorecard different interoperability Information Systems' related views can be compared and studied.

\section{APPLYING ISIMM}

The ISIMM is applied through a sequence of steps as illustrated in Figure 3 to assess the level of compliance and sophistication of a pairs, groups or clusters of interoperable information systems within a government organisation.

These steps constitute a recommended methodology for preparing and applying ISIMM.

Step 1: Set the organisations technical interoperability goals for the short, medium and long term in terms of the ISIMM maturity levels (e.g., long term target=level 4).

Step 2: Using Table 1 as a tool, asses the current state of technical interoperability of interoperable information system pairs, groups and clusters.

Assessment is performed by completing the assessment matrix (see Table 5) for each technical interoperability information system pair, group or sector. The minimum common set of attributes that make information systems interoperable needs to be marked with an ' $\mathrm{X}$ ' in Table 5 and summed per 
interoperability layer (i.e., D, S, C and P).

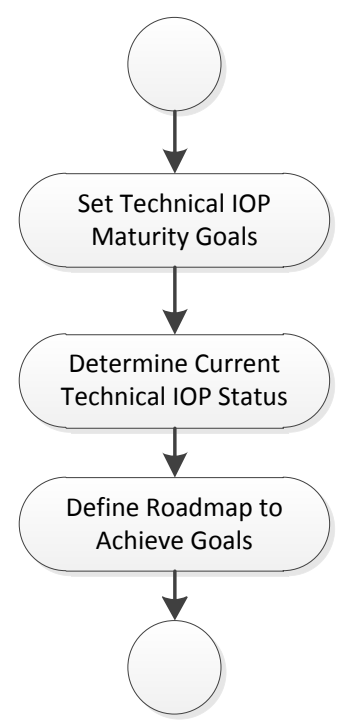

Figure 3: ISIMM application

Table 5 contains sample data for a technical interoperable pair of information systems. The pair obtained an overall rating of eight (8) which matched Level 3 as indicated in Tables 2. The eight (8) rating obtained is below the average compliance rating for Level 3 (see Table 2) which is nine (9), thus the level of technical interoperability attained is ' $3 \mathrm{~B}$ ' based on the measures of Table 3. Once all assessments are completed, they may be recorded on a scorecard as indicated in Table 4. Overall ratings for all assessments may be averaged to obtain an overall rating.

Step 3: Having established the current state and defined the desired state; develop a roadmap to achieve the set goals of step 1 .

Table 5. Information systems' interoperability maturity assessment matrix with example content

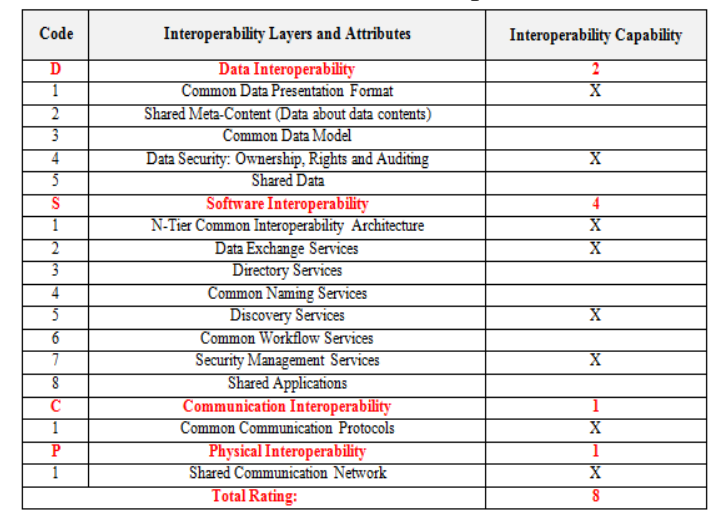

\section{CONCLUSION}

As discussed in Section 2, the LISI, C2, CMMI and GIMM models did not address the technical interoperability maturity and assessment needs to a satisfactory level of the Namibia Government Systems Architects. However, in this work, the ISIMM is envisaged that focuses on the more detailed technical aspects of interoperability maturity that allows data to be exchanged and shared within an information system environment at different compliance and sophistication levels. The ISIMM through the technical interoperability attributes defined, serves as basic technical standard to which public service organisations should strive to comply with.

The ISIMM with its assessment framework was tested with 24 information system pairs from seven (7) Namibia government organisations. The results from the tests suggested that the ISIMM and its assessment framework can be used as reference and benchmark for the different degrees of technical interoperability of information systems environments.

Expansion of interoperability attributes for each interoperability layer is underway, with the intention to improve assessment and referencing.

The current ISIMM model and its assessment framework could be made more inclusive by including aspects of organisational interoperability in particular business process interoperability that would allow end-to-end e-Government services.

\section{REFERENCES}

[1] United Nations Department for Economic and Social Affairs. (2008). United Nations E-Government Survey 2008: From E-Government to Connected Governance. New York: United Nations.

[2] Pardo, A. \& Burke, G.B. (2008). Improving Government Interoperability: A Capability Framework for Government Managers. Retrieved September 10, 2010, from University at Albany, Center for Technology in government Web site: http://www.ctg.albany.edu/publications/reports/im proving_government_interoperability

[3] Clark, T. \& Jones, R. (1999). Organisational Interoperability Maturity Model for C2., Command and Control Research and Technology Symposium. Retrieved March 7, 2011, from http://www.dodccrp.org/events/1999_CCRTS/pdf _files/track_5/049clark.pdf

[4] C4ISR Architectural Working Group. (1998). Levels of Information Systems Interoperability (LISI).Retrieved March 7, 2011, from http://www.defencelink.mil/nii/org/cio/i3/lisirpt.p df

[5] Carnegie Mellon, Software Engineering Institute. Capability Maturity Model Integration (CMMI). Retrieved November 18, 2010, from http://www.sei.cmu.edu/cmmi/general/index.html

[6] Sarantis, S., Charalabidis, Y., \& Psarras, J. (2008, May). Towards Standardising Interoperability Levels for Information Systems of Public Administration. Electronic Journal for e Commerce Tools and Applications (eJETA), Retrieved November 2, 2011, from http://www.ejeta.org/specialMay08-issue/ejeta-sp ecial-08may-5.pdf 
Stefanus van Staden is the Director of Systems Development and Implementation within the Office of the Prime Minister, Republic of Namibia. He received his M.Sc. degree in Information Technology from University of Namibia (UNAM) in 2012. He obtained a B.Tech. degree in Information Technology from University of South Africa (UNISA) in 2005 and a B.Sc. in Computer Science and Statistics from University of Namibia in 1990. His research interest include e-Government interoperability and intelligent online services.

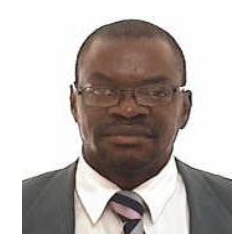

Jameson Mbale is a Senior Lecturer at the University of Namibia, in the Department of Computer Science. He received his $\mathrm{PhD}$ degree in Computer Science from Harbin Institute of Technology (HIT), China, in 2003. He obtained M.Sc. degree in Computer Science from Shanghai University, China, in 1996 and B.A., in Mathematics and Computer Science at University of Zambia in 1993 in Zambia. His research interest in network security, wireless networking, telecommunications and eGovernance. 